\title{
Massive soft tissue calcifications in a patient with end stage renal disease, complete resolved by Cinacalcet. A case report and review.
}

\author{
Fatemeh Espahbodi ${ }^{1}$, Maryam Mobini ${ }^{1}$, Ozra Akha ${ }^{1}$
}

${ }^{1}$ Department of Internal Medicine, Diabetes Research Center, Mazandaran University of Medical Sciences, Sari, Iran

\begin{abstract}
Metastatic calcification is the deposition of calcium salts in otherwise normal tissue as a severe complication of hemodialysis. It is associated with high levels of serum calcium and phosphorus product. We report a patient on hemodialysis who presented with progressively increasing, tumor-like calcinosis associated with muscle weakness. A 36-year-old male presented with multiple painful masses in different parts of his body that had been progressively increasing for ten months. We investigated and diagnosed him as secondary hyperparathyroidism and metastatic calcification; he was treated with cinacalcet. Given that the increase in parathormone and the onset of bone symptoms in the patient were not synchronous, the need for other markers to be reviewed for bone problems in this hemodialysis patient was confirmed.
\end{abstract}

Keywords: Hyperparathyroidism, cinacalcet, metastatic calcification

\section{Introduction}

Metastatic calcification (tumoral calcinosis) is an unusual and severe complication of dialysis (hemodialysis or peritoneal dialysis) characterized by large cystic or rubbery masses containing deposits of calcium and phosphate. Elevated levels of calcium-phosphorus products and severe secondary hyperparathyroidism are present in these patients. Common sites of involvement include blood vessels, the periarticular region, heart, lungs, kidneys, gastric mucosa, central nervous system, breasts, and eyes [1]. Secondary hyperparathyroidism is an important complication of chronic kidney disease (CKD) and end stage renal disease (ESRD). Elevated serum concentrations of parathyroid hormone (PTH) contribute to bone and cardiovascular disorders including myopathy, neuropathy, anemia, and pruritus. Current treatments consist of dietary phosphorus restriction, oral administration of intestinal phosphate binders, oral or intravenous calcitriol, oral calcimimetic agents (cinacalcet), and intensive hemodialysis with low calcium dialysate. Surgical excision of tumoral calcinosis, parathyroidectomy, and kidney transplantation are recommended for persistent or refractory cases $[1,2]$. Here we report a patient with ESRD complicated by metastatic calcification. The clinical manifestations, investigative findings, and treatments are reviewed in this paper.

\section{Case Report}

A 36-year-old man, a known case of ESRD under hemodialysis (three times/week, 4 hours/session), referred to a rheumatologist due to muscle weakness and joint pain experienced for the ten months prior to his referral. The patient complained of severe pain, swelling, and limited range of motion in his right shoulder and severe difficulty getting up from the ground. He denied any skin rash, oral ulcer, color changes in his extremities, dyspnea, or trauma. The patient had undergone kidney transplantation 9 years previously, but the transplant was rejected 3 years later. He received calcitriol $1.25 \mu \mathrm{g}$ three times per week. The amount of calcium in dialysate is $2.5 \mathrm{meq} / \mathrm{L}$.

In physical examinations, the patient was afebrile but had severe swelling and muscle atrophy in his right shoulder and erythematous swelling in his right wrist. The proximal muscular strength in his lower limbs was decreased (muscle could move only if the resistance of gravity was removed), but was intact in distal. In the patients' upper limbs, the proximal force was decreased in his left arm and was unreliable in his right side because of severe pain.

The following studies were performed to evaluate other possible causes or related illnesses: thyroid function test, parathyroid hormone, chest X-ray, echocardiography, blood test, and abdominopelvic sonography (Table

Personal non-commercial use only.Rheumatology Research Journal. Copyright (C) 2019. All rights reserved

*Corresponding Author: Maryam Mobini, Rheumatologist, Associate Professor, Diabetes Research Center, Mazandaran University of Medical Sciences, Sari, Iran. Email:mmobini50@yahoo.com, Tel/Fax : (+98)11333350672, (+98)1133264044.

Received: 12 June 2018 ; Accepted: 09 December 2018 
1). Radiographs of the shoulder, wrist, and hip showed widespread calcification with large cystic lesions and fluid levels in soft tissue. Due to the symptoms of lower limb weakness that was not justified by hyperparathyroidism alone, an MRI of the lumbosacral vertebra was requested. EMG/NCV revealed the myogenic noninflammatory process. To assess hyperparathyroidism (to rule out adenoma), a Sestamibi scan was requested, but showed no problems. Bone densitometry by DEXA showed low $\mathrm{T}$ and $\mathrm{Z}$ scores in the spine $(-2.4$ and -2.3$)$ and femur $(-2.4$ and $-1.9)$. Due to high levels of calcium - phosphorus product, calcitriol was discontinued and Sevelamer $(800 \mathrm{mg}$
TDS) (as a phosphate binder) was started, but because of sustained hyperparathyroidism, Cinacalcet (Mimpara) 30 $\mathrm{mg}$ daily was added. Draining the bulky shoulder deposits by surgery was considered, but during the three months after treatment, the patient felt healed, his shoulder range of motion was increased, and his muscle weakness markedly declined. Nine months later, joint pain and swelling had disappeared, and muscle force was totally recovered. Laboratory data at this time included: calcium:8.87 mg/dl, phosphur:5.37 mg/dl, Alb:4.3, PTH: 378 pg/ml (10.4-67), ESR: $36 \mathrm{~mm} / \mathrm{h}, \mathrm{CRP}: 42.6 \mathrm{mg} / \mathrm{l}$ (up to 10 ).

Table 1. Laboratory tests and imaging results of the patient

\begin{tabular}{|c|c|}
\hline Laboratory tests & Results \\
\hline $\mathrm{CBC}$ & WBC: 10000, Hg: 13.5 g/dl, Plt: 304000 \\
\hline Acute phase reactants & ESR: $83 \mathrm{~mm} / \mathrm{h}, \mathrm{CRP}: 91 \mathrm{mg} / \mathrm{l}$ (up to 10 ) \\
\hline Biochemical tests & $\begin{array}{l}\text { FBS: } 89 \text { mg/dl, cholesterol: } 196 \text { mg/dl, triglyceride: } 163 \mathrm{mg} / \mathrm{dl} \text {, creatinine: } 8.5 \\
\text { mg/dl, ALT: } 30 \text { IU/L, albumin: } 4.1 \mathrm{~g} / \mathrm{dl} \text {, uric acid: } 4.6 \mathrm{mg} / \mathrm{dl} \text {, calcium: } 10.2 \\
\text { mg/dl, phosphur: } 7 \mathrm{mg} / \mathrm{dl}\end{array}$ \\
\hline Rheumatologic tests & $\begin{array}{l}\text { RF: neg, ANA: neg, AntiCCP: neg, HLA B 27: negative, C and P ANCA: neg, } \\
\text { CPK:31 U/L, LDH:261 IU/L(ULN:480), aldolase: 7.6 U/L(ULN:7.6) }\end{array}$ \\
\hline Endocrinology & TSH: 1.38 ng/ml (0.46), PTH:604 pg/ml (ULN:87) \\
\hline Viral markers & Hbs Ag: neg, HCV Ab: pos, HIV Ab: neg \\
\hline Imaging & $\begin{array}{c}\text { Chest Xray: NL } \\
\text { Abdominopelvic sonography: NL } \\
\text { Echocardiography: NL } \\
\text { BMD: secondry osteoporosis }\end{array}$ \\
\hline
\end{tabular}

In First visit

Three month later

Two years later
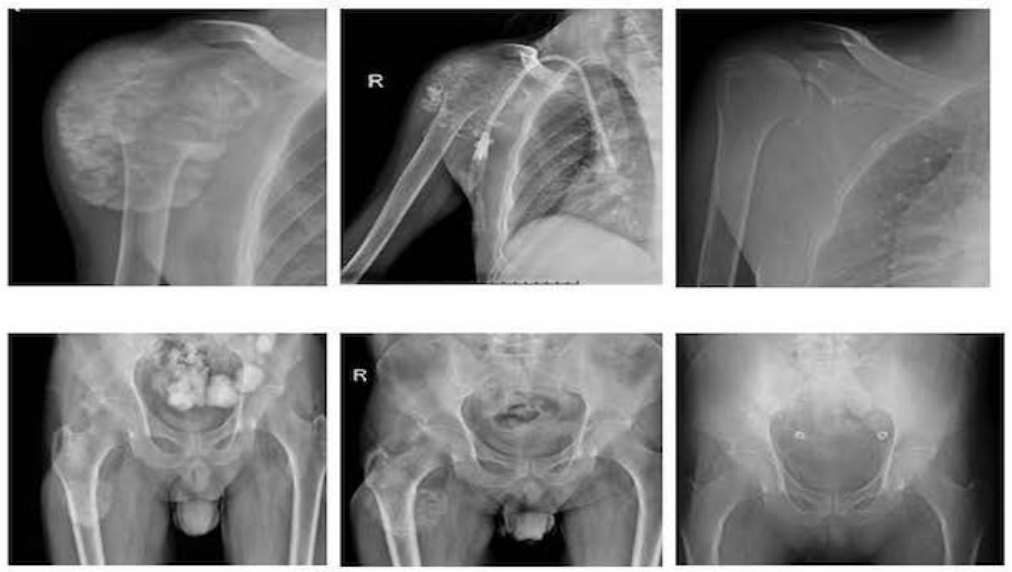

Figure 1. Changes in calcification during treatment with cinacalcet. 


\section{Discussion}

The diagnosis of metastatic calcification is based on a combination of clinical manifestation, laboratory and radiographic changes. Our patient presented with severe joint pain, muscle weakness, massive tumoral calcinosis, hyperphosphatemia, and hyperparathyroidism. There were some challenges in the diagnosis and management of this patient. Acute phase reactants (ESR and CRP) were high, so we decided to evaluate the patient for infectious, malignant, and rheumatologic disorders (Table 1). Muscle weakness may be due to different disorders such as inflammatory or metabolic and endocrine reasons. Muscle enzymes and $\mathrm{EMG} / \mathrm{NCV}$ did not match with inflammatory muscle disorder. The bone and joint problems of the patient began 9 months prior to his referral. The diagnosis was delayed due to the slow and progressive development of manifestations.

In kidney dysfunction, secondary hyperparathyroidism and progressive parathyroid gland hyperplasia develop in disturbances of pathophysiologic regulation of $\mathrm{Ca}, \mathrm{P}$, and vitamin D metabolism and fibroblast growth factor 23 (FGF23) [3]. Cinacalcet is a calcimimetic that shifts the Ca-PTH concentration-response curves to the left, so it can reduce the set point for Ca-related $\mathrm{PTH}$ secretion, decrease secretion of PTH, and diminish serum $\mathrm{Ca}$ concentrations.

The efficacy of cinacalcet in reducing circulating levels of $\mathrm{PTH}, \mathrm{Ca}, \mathrm{P}$, and $\mathrm{Ca} \times \mathrm{P}$ product in dialysis patients with secondary hyperparathyroidism [4]. In a study on 59 kid- ney transplant recipients with hyperparathyroidism, parathyroidectomy was compared with cinacalcet by changes in calcium and phosphorus and PTH. The study demonstrated that correction of severe hyperparathyroidism was similar in both surgical and cinacalcet groups [5]. The effect of cinacalcet was shown in a CKD patient with secondary hyperparathyroidism despite subtotal parathyroidectomy [6]. In the current patient, the beneficial and fast effects of cinacalcet were shown in clinical, laboratory and radiological symptoms.

It seems that there is no significant correlation between PTH and bone disorder in this patient, and hyperphosphatemic therapy in addition to reducing PTH levels have been effective in improving the patient's health. The patient's bone changes had begun before PTH was increased; this confirms the need for the review of other markers for bone problems in hemodialysis patients. PTH may not be a sensitive marker in some patients.

In conclusion, this observation supports the concept that calcimimetic cinacalcet can be used in patients with secondary hyperparathyroidism. It is suggested that more attention should be paid to calcium and phosphorus levels in order to diagnose and treatment of bone problems in dialysis patients.

\section{Conflict of Interest}

The authors declare no conflicts of interest 


\section{References}

1. Maioli ME, Delfino VDA, Guerra A, Kunii LF, Frange RFN. Reversal of uremic tumoral calcinosis by optimization of clinical treatment of bone and mineral metabolism disorder. J Bras Nefrol. 2017;39(2):217-9. doi: 10.5935/0101-2800.20170037.

2. Block GA, Bushinsky DA, Cheng S, Cunningham J, Dehmel B, Drueke TB, et al. Effect of Etelcalcetide vs Cinacalcet on Serum Parathyroid Hormone in Patients Receiving Hemodialysis with Secondary Hyperparathyroidism: A Randomized Clinical Trial. Jama. 2017;317(2):156-64. doi: 10.1001/jama.2016.19468.

3. Cunningham J, Locatelli F, Rosriguez M: Secondary hyperparathyroidism: Pathogenesis, disease progression, and therapeutic options. Clin J Am Soc Nephrol.2011; 6(4): 913-921. doi: 10.2215/cjn.06040710.
4. Bover J, Urena P, Ruiz-Garcia C, daSilva I, Lescano P, del Carpio J, et al. Clinical and Practical Use of Calcimimetics in Dialysis Patients with Secondary Hyperparathyroidism. Clin J Am Soc Nephrol. 2016;11(1):161-74. doi: 10.2215/ cjn.01760215.

5. Soliman AR, Maamoun HA, Soliman MA, Darwish H, Elbanna E. Cinacalcet versus Parathyroidectomy in the Treatment of Secondary Hyperparathyroidism Post Renal Transplantation. Rom J Intern Med. 2016;54(3):184-9. doi: 10.1515/rjim-2016-0027.

6. Staszkow M, Wojtaszek E, Zebrowski P, Matuszkiewicz-Rowinska J. Massive soft tissue calcifications in severe hyperparathyroidism secondary to end-stage renal disease. Pol Arch Med Wewn. 2013;123(4):191-2. doi: 10.20452/pamw.1690. 